\title{
Subcutaneous gentamicin injection around the cuff in treatment of resistant exit site infection in peritoneal dialysis patients: a pilot study
}

This article was published in the following Dove Press journal:

Therapeutics and Clinical Risk Management

20 July 2017

Number of times this article has been viewed

\author{
Oguzhan Sıtkı Dizdar' \\ Ozerhan Ozer ${ }^{2}$ \\ Selahattin Erdem ${ }^{2}$ \\ Ali Ihsan Gunal ${ }^{3}$ \\ 'Department of Internal Medicine and \\ Clinical Nutrition, Kayseri Training and \\ Research Hospital, Kayseri, Turkey; \\ ${ }^{2}$ Department of Internal Medicine, \\ Kayseri Training and Research \\ Hospital, Kayseri, Turkey; ${ }^{3}$ Department \\ of Internal Medicine Division of \\ Nephrology, Kayseri Training and \\ Research Hospital, Kayseri, Turkey
}

Background/purpose: One of the most common complications of the peritoneal dialysis (PD) is the infection of the exit site of the peritoneal catheter. The aim of the present study was to evaluate the efficacy of the subcutaneous gentamicin injection around the cuff as a part of routine treatment of the resistant exit site infection (ESI).

Methods: If the exit site remains infected after a 2-week systemic antibiotics treatment, it is defined as resistant ESI. In these cases, systemic antibiotics were discontinued and a subcutaneous 40-mg gentamicin injection was administered around the external cuff of the PD catheter every 3 days. A total of three or four injections were given to each patient.

Results: A subcutaneous gentamicin injection was administered around the cuff in thirteen patients for the treatment of resistant ESI over a 2-year period. The median follow-up time in cured patients was 12 months. Eleven of the thirteen patients had been apparently cured of their resistant ESI, with no recurrence. None of the patients had a gentamicin-resistant species. Subcutaneous gentamicin-related adverse effect was not observed in any patient.

Conclusion: Subcutaneous gentamicin injection around the cuff is a well-tolerated and effective strategy for treating resistant ESI. To gain widespread approval of this therapy and reach a consensus about ESI management, additional studies are needed.

Keywords: peritoneal dialysis, subcutaneous gentamicin, local treatment, catheter, efficacy

\section{Introduction}

Peritoneal dialysis (PD) has proved to be an effective therapeutic option for patients with end-stage renal disease. However, despite technical improvements, one of the most common complications of the PD is the infection of the exit site of the peritoneal catheter. Exit-site infections (ESIs) are responsible for $20 \%$ of peritoneal infections, provoke $20 \%$ of catheter removals, ${ }^{1}$ and are implicated in the transfer to hemodialysis (HD) in $15 \%-20 \%$ of patients. ${ }^{2}$ Therefore, there is a need to prevent and effectively treat ESIs.

The most common cause of ESI and peritonitis is gram-positive organisms., Staphylococcus aureus is one of the major causes of ESI and peritonitis, which are often difficult to treat due to the virulence of the organism. ${ }^{5}$ Some $S$. aureus organisms are resistant to antibiotics (particularly methicillin-resistant $S$. aureus strains); vancomycin is the treatment of choice for serious infections with these organisms. ${ }^{6}$ Recently, ciprofloxacin and other quinolones have been introduced but, unfortunately, the use of such drugs to treat $S$. aureus infection has been associated with rapid and extensive emergence of resistance. ${ }^{7}$ Similarly, the use of rifampicin
Correspondence: Oguzhan Sıtkı Dizdar Kayseri Training and Research Hospital, Atatürk Avenue Hastane street No 78, Postal Code: 38010 , Kayseri, Turkey Tel +90352 3368884

Fax+903523368857

Email osdizdar@gmail.com 
has been associated with serious side effects and resistance even when combined with other agents. ${ }^{8}$ Carriage of $S$. aureus in the nares, hands, or groin has also been linked to increased risk of ESI. ${ }^{9}$

Examples of local therapy are usually in the form of topical creams and these local therapies are mostly used to prevent the development of ESI. ESI caused by $S$. aureus infection can be prevented by the daily pericatheteral application of mupirocin ointment. ${ }^{10-14}$ The common use of other antibiotics such as topical ciprofloxacin, which reduces ESIs caused by $S$. aureus and Pseudomonas aeruginosa, as well as the topical use of gentamicin are to prevent ESI.

International Society for Peritoneal Dialysis guidelines recommend the use of one to two systemic antibiotics in the case of ESI. ${ }^{15}$ But there are no data or recommendations about any topical or local treatment of ESI, especially resistant ESI. Whether the use of subcutaneous gentamicin injection around the cuff in resistant ESI will enhance the cure rate is unknown. Therefore, we hypothesized that a subcutaneous gentamicin injection around the cuff is a good adjunctive in the treatment of resistant ESI of PD patients. The aim of the present study was to evaluate the efficacy of this injection around the cuff as a part of routine treatment of the resistant ESI.

\section{Materials and methods}

This is a single-center prospective analysis of resistant ESI in PD patients from March 2013 to February 2016 at the Nephrology Clinic of Kayseri Training and Research Hospital. All patients $\geq 18$ years of age were included in the study. The exclusion criteria were 1) allergy to gentamicin, 2) patients not accepting this procedure, 3 ) patients with tunnel infection and peritonitis, 4) fungal infection, and 5) pregnant women. All procedures involving human participants were performed in accordance with the ethical standards of the
Institutional Research Committee of Kayseri Training and Research Hospital and with those of the Helsinki Declaration. A written informed consent was obtained from all subjects included in the present study.

All PD patients were clinically monitored for signs of ESI at their regular clinic visits (every 6-8 weeks). Local application of mupirocin or povidone iodine for every 2-3 days was part of routine exit care in our center.

ESI was defined as the presence of purulent discharge, erythema, and/or positive bacterial culture from the exit site (Figure 1). Redness or skin induration may or may not represent an infection. Formation of a crust around the exit may not indicate infection. Resolution of the ESI was defined by the absence of any clinical signs for ESI and a negative culture. A scoring system developed by Schafer F et al was used to monitor ESI. ${ }^{16}$ There were five parameters (swelling, crust, redness, pain, and drainage) that suggested ESI in this scoring system and ESI should be assumed with an exit-site score of four or greater. Purulent drainage, even if alone, is sufficient to indicate infection. Our standard treatment protocol for ESI consisted of oral ciprofloxacin or sodium fusidate monotherapy. Patients were advised to take ciprofloxacin at least for 2-4 $\mathrm{h}$ apart from any phosphate binders. Gram stain and the culture of the drainage or the microbiological history of a preceding ESI can help in the therapy. Antibiotic treatment is continued until the exit site appears healthy. Two weeks is the minimum period, but treatment for 3 weeks is most likely necessary for $P$. aeruginosa infections. If the exit site remains infected after 2 weeks of systemic antibiotics, it is defined as resistant ESI.

We included and followed only resistant ESI cases during the study period. In case of resistant ESI, systemic antibiotics were discontinued and a subcutaneous 40-mg gentamicin injection was administered around the external cuff of the PD catheter every 3 days (Figure 2). A total of three or four injections were given to each patient.
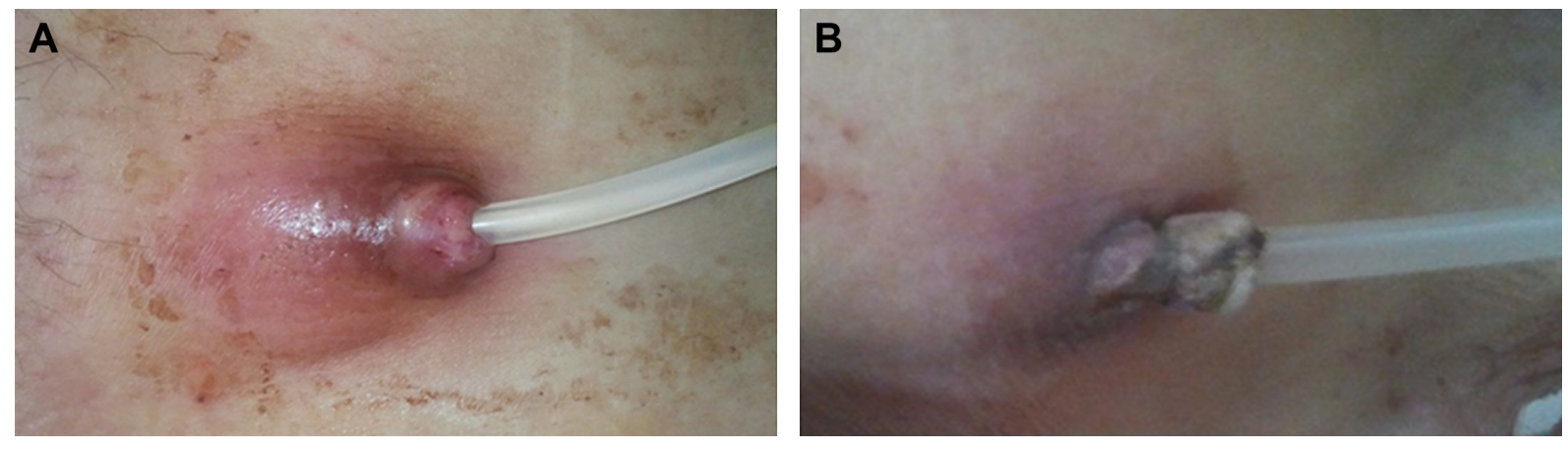

Figure I An example of peritoneal dialysis catheter exit site infection (ESI).

Notes: (A) Before subcutaneous gentamicin injection into the pericatheteral area and (B) after subcutaneous gentamicin injection into the pericatheteral area. 


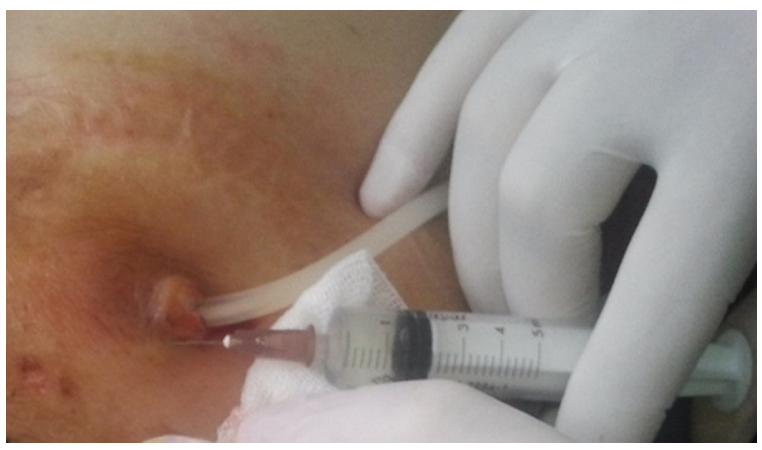

Figure 2 Application of subcutaneous gentamicin injection into the pericatheteral area in a PD patient with ESI.

Abbreviations: ESI, exit site infection; PD, peritoneal dialysis.

\section{Statistical analysis} normality and the homogeneity of the data were evaluated by the Shapiro-Wilk test and the Levene test, respectively. All SPSS, Chicago, IL, USA). $P<0.05$ was considered statistically significant.

\section{Results}

Subcutaneous gentamicin injection was administered around the cuff in thirteen patients for the treatment of resistant ESI over a 2-year period. The demographic data and the clinical characteristics of the patients are summarized in Table 1. Eight patients were male and five patients were female with a median age of 54 years (range 33-78).

Eleven of the thirteen patients were apparently cured of their ESI, with no recurrence (Table 2). The median follow-up time in the cured patients was 12 months (range 8-24). study population

\begin{tabular}{ll}
\hline Variable & $\mathbf{n}=\mathbf{1 3}$ \\
\hline Age, median (range) & $54(33-78)$ \\
Gender, $\mathbf{n}(\%)$ & \\
Male & $8(61)$ \\
Female & $5(39)$ \\
Duration of CAPD, year, median (range) & $4.5(\mathrm{I}-8)$ \\
Etiology of renal failure & \\
Diabetic nephropathy & 3 \\
Hypertensive nephropathy & 3 \\
Chronic pyelonephritis & 2 \\
Nephrolithiasis & $\mathrm{I}$ \\
Others/unknown & 4 \\
Refractory ESI history, $\mathrm{n}(\%)$ & $2(15)$ \\
Patients with cure, $\mathrm{n}(\%)$ & $\mathrm{II}(85)$ \\
Patients with recurrence, $\mathrm{n}(\%)$ & $\mathrm{I}(7.5)$ \\
Catheter removal, $\mathrm{n}(\%)$ & $\mathrm{I}(7.5)$ \\
\hline
\end{tabular}

Abbreviations: CAPD, continuous ambulatory peritoneal dialysis; ESI, exit site infection.
Data were expressed as the median (including range). The calculations used the SPSS statistical package (version 15.0;

Table I Demographic data and clinical characteristics of the

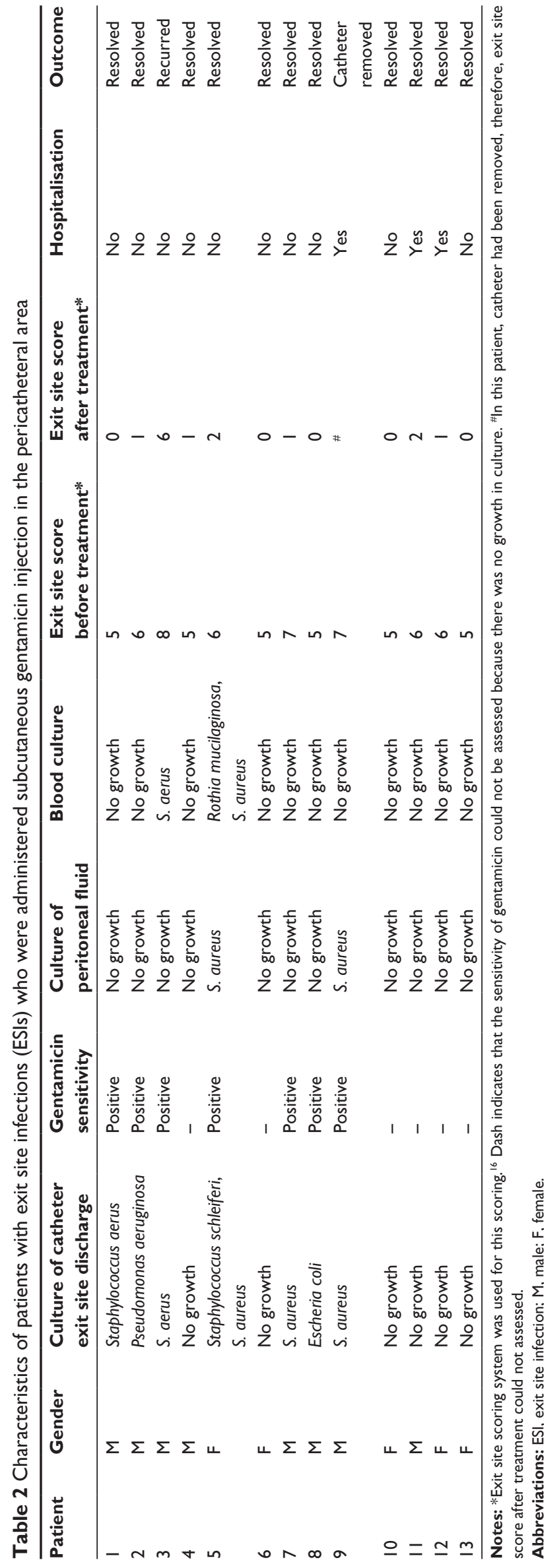

Therapeutics and Clinical Risk Management 2017:13 
One resistant ESI episode resulted in catheter removal. The catheter was removed in this patient because of the concomitant severe peritonitis. Recurrence of ESI occurred in only one case. Five patients (39\%) had developed ESI due to $S$. aureus. The other cases were due to PA and Escheria coli. We showed differences before and after gentamicin treatment according to the exit-site scoring system in Table 2. None of the patients had a gentamicin-resistant species. Subcutaneous gentamicin-related adverse effect was not observed in any patient.

\section{Discussion}

Peritoneal dialysis is an effective and lower-cost form of renal replacement therapy. It can achieve a similar, or even better, mortality rate than hemodialysis does, especially in the first 2 years of dialysis. ${ }^{17}$ Catheter-related infective complications (ESI, tunnel infection, and peritonitis) are the most frequent causes of method dropout in PD and are also a significant cause of morbidity, hospitalization, catheter removal, and even death. ${ }^{18-20}$ Patients with ESI also present a higher incidence of peritonitis. ${ }^{21}$ Therefore, treatment of catheter-related infections in patients undergoing PD is crucial to the success of this type of renal replacement therapy. Although, some advances have been made in the treatment of this complication with topical antiseptics and systemic antibiotic therapy, outcomes continue to remain poor and lead to catheter removal. While antibiotic cream/ ointment at the exit site lowers the risk of infection, the use increases the resistance of different organisms and therefore alternate approaches are preferable. The use of a therapeutic concentration of antibiotics is an important precaution against wound infection or catheter ESI. Gentamicin is a widely used antibiotic, having antibacterial activity against aerobic gram-negative bacteria including members of the families Enterobacteriaceae and Pseudomonadaceae, which includes the human pathogen PA and the gram-positive organism $S$. aureus. Pathogens most often obtained in the cultures of specimens from the catheter exit site of PD patients belong to this group. The reason we chose subcutaneous gentamicin for resistant ESI treatment was that microorganisms that commonly isolated from the exit site were susceptible to gentamicin. One major concern in the treatment of ESI is that orally or systematically administered antibiotics achieve effective concentrations in the affected tissue (around the cuff). Because of low success rate, we can speculate that these antibiotics do not reach an effective dose in or around the cuff. Local application of antibiotics has been shown to prevent ESI, but there is little information about their use of treatment of ESI. ${ }^{13,15}$ In addition, no study has proven that local injection of gentamicin results in significantly lower rates of ESI in PD patients.

This study reviewed thirteen patients with resistant ESI who had undergone subcutaneous gentamicin injection around the cuff. According to our knowledge, this is the first study looking into the role of this procedure for the treatment of resistant ESI in PD patients. Our analysis suggests that subcutaneous gentamicin injection around the cuff can be an important option in the treatment of PD patients with resistant ESI. The overall cure rate of $85 \%$ in our population was better than previously reported cure rates with different treatment methods. ${ }^{22-24}$ Therefore, subcutaneous gentamicin injection around the cuff is feasible for eradicating resistant ESI, as evidenced by the low incidence of recurrence of ESI after the procedure. Only one of thirteen patients had developed a recurrence of ESI within the follow-up duration. These results provide a new knowledge that subcutaneous gentamicin may be an effective treatment in resistant ESI.

ESI with pseudomonas is recognized as a major complication of $\mathrm{PD}$, with high risk of catheter loss due to refractory/ recurrent infection or peritonitis. There is remarkably little literature about treatment outcomes in patients with pseudomonas ESI and there is no standard treatment protocol. The reported cure rate of pseudomonas ESI with different systemic antibiotic treatment regimens ranges from $42 \%$ to $83 \% .^{22,23,25}$ In our study, only one patient had pseudomonas ESI and he was successfully treated with subcutaneous gentamicin. In Burkhalter et al's study, in total, in only $50 \%$ of the patients was pseudomonas ESI successfully treated with topical gentamicin and oral ciprofloxacin, and their study confirmed that pseudomonas ESI is an important cause of PD technique failure ${ }^{24}$ and that the application of the topical gentamicin cream is not an effective method in the treatment of pseudomonas ESI. The possible reason for this treatment failure may be that antibiotics are not sufficiently diffused into the cuff. But there is no information about subcutaneous gentamicin injection in the treatment of these patients. We speculate that this procedure may provide additional benefits.

The topical application of antibiotics might also be useful in preventing ESI. Montenegro et al reported that ciprofloxacin otologic solution applied daily to the exit site could significantly reduce the incidence of ESI caused by $S$. aureus and $P$. aeruginosa. ${ }^{26}$ Bernardini et al showed that the daily application of the gentamicin cream to the exit site was effective in preventing the development of ESI by both gram-positive and gram-negative organisms. ${ }^{27}$ But the 
topical gentamicin cream did not show similar success rates in the treatment of pseudomonas ESI in Burkhalter et al's study and the success rate of this treatment is not higher than $50 \%-60 \%{ }^{24}$ The biofilm formed by bacteria lowers the efficacy of the topical gentamicin in the treatment of ESI and systemic antibiotics cannot probably reach sufficient concentrations in the pericatheteral area in PD patients with ESI. In contrast to topical gentamicin cream application, our results showed that subcutaneous gentamicin injection around the cuff had a higher success rate in the treatment of ESI. Furthermore, a significant concern with the prolonged use of any antibiotic is the development of resistance. But our treatment could reduce this prolonged use of antibiotic in these patients. An exposed cuff may cause resistant infection and the tissue surrounding the catheter and the cuff is debrided, and the cuff is removed in these cases. It may not be necessary to implement this surgical procedure with our treatment.

The application of subcutaneous gentamicin at the catheter exit site is considered to be a very safe method. No significant adverse effects were reported in the present study. But this study did not measure the systematic absorption of subcutaneous gentamicin and the level of serum gentamicin Although we did not see any adverse effect or ototoxicity related to subcutaneous gentamicin, future studies should consider measuring the serum gentamicin level.

There were some limitations in our study. We did not have a control group without the use of subcutaneous gentamicin and the study was not blinded in any way. Other limitations were that it was a single-center study and the number of patients in the study was small.

\section{Conclusion}

Subcutaneous gentamicin injection around the cuff for treating resistant ESI is a well-tolerated and effective strategy. Probably, it can significantly reduce morbidity, catheter loss, and transfer to hemodialysis as well as recurrence of ESI. We should further study the efficacy of subcutaneous gentamicin injection in the treatment of ESIs caused by different organisms. If a reduction of recurrence of infections related to this procedure could be confirmed, it would present a major advantage, since it would imply a reduction of the high morbidity associated with such infections and of the frequent need for catheter removals. To gain widespread approval of this therapy and reach a consensus about resistant ESI management, additional studies are needed. We recommend that this new treatment method be applied to patients who have resistant and frequent catheter infections.

\section{Acknowledgments}

We thank the Peritoneal Dialysis Unit of Kayseri Training and Research Hospital for kind help. The study was not financially supported.

\section{Disclosure}

The authors report no conflicts of interest in this work.

\section{References}

1. Montenegro J. Prevention and treatment of peritoneal catheter exit-site infection. Nefrología. 1999;19:502-507.

2. Kolesnyk I, Dekker FW, Boeschoten EW, Krediet RT. Time dependent reasons for peritoneal dialysis technique failure and mortality. Perit Dial Int. 2010;30(2):170-177.

3. Troidle L, Gorban-Brennan N, Kliger A, Finkelstein F. Differing outcomes of gram-positive and gram-negative peritonitis. Am J Kidney Dis. 1998;32(4):623-628.

4. Ward A, Campoli-Richards DM. Mupirocin. A review of its antibacterial activity, pharmacokinetic properties and therapeutic use. Drugs. 1986;32:425-444.

5. Zimmerman SW, O'Brien M, Wiedenhoeft F, Johnson C. Staphylococcus aureus peritoneal catheter-related infections: a cause of catheter loss and peritonitis. Perit Dial Int. 1988;8:191-194.

6. Eykyn SJ. Staphylococcal sepsis. The changing pattern of disease and therapy. Lancet. 1988;1(8577):100-103.

7. Cafferkey MT. Therapy for staphylococcal infection. Curr Opin Infect Dis. 1991;4:757-764.

8. [No authors listed]. Revised guidelines for the control of epidemic methicillin-resistant Staphylococcus aureus. J Hosp Infect. 1990;16(4): 351-377.

9. Turner K, Uttley L, Scrimgeour A, Mckewan A, Gokal R. Natural history of Staphylococcal aureus nasal carriage and its relationship to exit site infection. Perit Dial Int. 1998;18(3):271-273.

10. Wong SS, Chu KH, Cheuk A, et al. Prophylaxis against gram-positive organisms causing exit-site infection and peritonitis in continuous ambulatory peritoneal dialysis patients by applying mupirocin ointment at the catheter exit site. Perit Dial Int. 2003;23 (Suppl 2):153-158.

11. Bernardini J, Piraino B, Holley J, Johnston JR, Lutes R. A randomized trial of Staphylococcus aureus prophylaxis in peritoneal dialysis patients: mupirocin calcium ointment $2 \%$ applied to the exit site versus cyclic oral rifampicin. Am J Kidney Dis. 1996;27:695-700.

12. Lim CT, Wong KS, Foo MW. The impact of topical mupirocin on peritoneal dialysis infection rates in Singapore General Hospital. Nephrol Dial Transplant. 2005;20(8):1702-1706.

13. Uttley L, Vardhan A, Mahajan S, Smart B, Hutchison A, Gokal R. Decrease in infections with the introduction of mupirocin cream at the peritoneal dialysis catheter exit site. J Nephrol. 2004;17(2): $242-245$.

14. Mahajan S, Tiwari SC, Kalra V, et al. Effect of local mupirocin application on exit site infection and peritonitis in an Indian peritoneal dialysis population. Perit Dial Int. 2005;25(5):473-477.

15. Li PK, Szeto CC, Piraino B, et al. Peritoneal dialysis-related infections recommendations: 2010 update. Perit Dial Int. 2010;30(4):393-423.

16. Schaefer F, Klaus G, Muller-Wiefel DE, Mehls O. Intermittent versus continuous intraperitoneal glycopeptide/ceftazidime treatment in children with peritoneal dialysis-associated peritonitis. The Mid-European Pediatric Peritoneal Dialysis Study Group (MEPPS). J Am Soc Nephrol. 1999;10(1):136-145.

17. Fenton SS, Schaubel DE, Desmeules M, et al. Hemodialysis versus peritoneal dialysis: a comparison of adjusted mortality rates. Am J Kidney Dis. 1997;30(3):334-342.

18. Fried L, Abidi S, Bernardini J, Johnston JR, Piraino B. Hospitalization in peritoneal dialysis patients. Am J Kidney Dis. 1999;33(5):927-933. 
19. Golper TA, Brier ME, Bunke M, et al. Risk factors for peritonitis in long-term peritoneal dialysis: the Network 9 peritonitis and catheter survival studies. Am J Kidney Dis. 1996;28(3):428-436.

20. Fried LF, Bernardini J, Johnston JR, Piraino B. Peritonitis influences mortality in peritoneal dialysis patients. J Am Soc Nephrol. 1996;7(10): 2176-2182.

21. Abraham G, Savin E, Blake P, Dombros N, Símbolos K. Natural history of exit-site infection in patients on CAPD. Perit Dial Bull. 1988; 3:211-216.

22. Lo CY, Chu WL, Wan KM, et al. Pseudomonas exit-site infections in CAPD patients: evolution and outcome of treatment. Perit Dial Int. 1998; 18(6):637-640.

23. Szabo T, Siccion Z, Izatt S, Vas SI, Bargman J, Oreopoulos DG. Outcome of Pseudomonas aeruginosa exit-site and tunnel infections: a single center's experience. Adv Perit Dial. 1999;15:209-212.
24. Burkhalter F, Clemenger M, Haddoub SS, McGrory J, Hisole N, Brown E. Pseudomonas exit-site infection: treatment outcomes with topical gentamicin in addition to systemic antibiotics. Clin Kidney J. 2015;8(6): 781-784.

25. Kazmi HR, Raffone FD, Kliger AS, Finkelstein FO. Pseudomonas exit site infections in continuous ambulatory peritoneal dialysis patients. J Am Soc Nephrol. 1992;2:1498-1501.

26. Montenegro J, Saracho R, Aguirre R, Martinez I, Iribar I, Ocharan J. Exit-site care with ciprofloxacin otologic solution prevents polyurethane catheter infection in peritoneal dialysis patients. Perit Dial Int. 2000; 20(2):209-214.

27. Bernardini J, Bender F, Florio T, et al. Randomized, double-blind trial of antibiotic exit site cream for prevention of exit site infection in peritoneal dialysis patients. J Am Soc Nephrol. 2005;16(2):539-545.
Therapeutics and Clinical Risk Management

\section{Publish your work in this journal}

Therapeutics and Clinical Risk Management is an international, peerreviewed journal of clinical therapeutics and risk management, focusing on concise rapid reporting of clinical studies in all therapeutic areas, outcomes, safety, and programs for the effective, safe, and sustained use of medicines. This journal is indexed on PubMed Central, CAS,

\section{Dovepress}

EMBase, Scopus and the Elsevier Bibliographic databases. The manuscript management system is completely online and includes a very quick and fair peer-review system, which is all easy to use. Visit http://www.dovepress.com/testimonials.php to read real quotes from published authors.

Submit your manuscript here: http://www.dovepress.com/therapeutics-and-clinical-risk-management-journal 